\title{
EchoGéo
}

$9 \mid 2009$

L'Asie centrale : le temps des recompositions

\section{Introduction au dossier "L'Asie centrale »}

Renaissance et recomposition d'un espace régional oublié

\section{Alain Cariou}

\section{CpenEdition}

\section{Journals}

Édition électronique

URL : https://journals.openedition.org/echogeo/11228

DOI : $10.4000 /$ echogeo. 11228

ISSN : 1963-1197

Éditeur

Pôle de recherche pour l'organisation et la diffusion de l'information géographique (CNRS UMR 8586)

Référence électronique

Alain Cariou, «Introduction au dossier «L'Asie centrale » », EchoGéo [En ligne], 9 | 2009, mis en ligne le 04 juillet 2009, consulté le 31 juillet 2021. URL : http://journals.openedition.org/echogeo/11228 ; DOI : https://doi.org/10.4000/echogeo.11228

Ce document a été généré automatiquement le 31 juillet 2021.

EchoGéo est mis à disposition selon les termes de la licence Creative Commons Attribution - Pas d'Utilisation Commerciale - Pas de Modification 4.0 International (CC BY-NC-ND) 


\title{
Introduction au dossier « L'Asie centrale »
}

\author{
Renaissance et recomposition d'un espace régional oublié
}

\author{
Alain Cariou
}

1 Avec les bouleversements géopolitiques qui affectent le continent asiatique depuis deux décennies l'Asie centrale n'est plus tout à fait cet espace géographique incertain écarté de la communauté internationale, voire même oublié des atlas dont les cartes étaient généralement centrées sur les territoires voisins plus lisibles et médiatiques du MoyenOrient, de l'Inde, de la Chine ou de la Russie. Constituée par les cinq ex-républiques socialistes soviétiques du Kazakhstan, du Kirghizstan, de l'Ouzbékistan, du Tadjikistan et du Turkménistan auxquelles il faut ajouter le Xinjiang, région autonome ouigoure de la République populaire de Chine, cette entité régionale centrasiatique à l'unité pourtant incontestable sur le plan géographique et culturel avait même disparu corps et biens de la géographie classique durant le $\mathrm{XX}^{\mathrm{e}}$ siècle. En dépit d'une forte cohérence humaine fondée sur la communauté des langues turciques, une religion partagée, l'islam, et un passé commun forgé autour de la culture turco-iranienne, cette Asie centrale a été marginalisée et découpée sous l'effet de la poussée impérialiste russe et chinoise au XIX ${ }^{e}$ siècle puis par l'avènement des deux géants communistes, l'URSS et la République populaire de Chine. Eclipsé par la tutelle de ces grandes puissances, notre ensemble régional devenait un "hinterland amorphe» (Aubin, 1990), une marge d'empires qui n'avait guère de résonance et de signification dans le concert mondial. Cet enfermement politique a culminé du temps de la Guerre froide et lors du conflit sino-soviétique, période où la région transformée en glacis militaire est restée isolée des regards extérieurs par des frontières hautement verrouillées et surveillées. Mais cette marginalisation découle aussi de facteurs géographiques. Avec un peu moins de 80 millions d'habitants pour 5,6 millions de $\mathrm{km}^{2}$ on tient généralement l'Asie centrale pour un territoire du vide, souvent mise en opposition avec les puissances démographiques environnantes comme la Chine orientale et l'Inde. Au peuplement lacunaire éparpillé en archipels parmi l'immensité des steppes et des déserts s'ajoute l'effet de l'enclavement. La position continentale au cœur de l'Eurasie n'a guère contribué à faire de la région un des hauts lieux des échanges mondiaux, l'Asie centrale 
constituant en effet le plus vaste ensemble d'Etats enclavés du monde (Cariou, 2007). L'enclavement régional reste encore un handicap à vaincre.

2 Toutefois la région n'a pas toujours été cet «angle mort " géographique, et son passé nous révèle qu'elle a bien au contraire été un carrefour, une terre de passage et d'échanges entre la Chine et l'Europe. De l'Antiquité au Moyen Age l'Asie centrale a participé à l'essor des économies-monde, au sens braudélien du terme, grâce à l'écheveau des pistes caravanières et aux illustres cités marchandes de la Route de la soie (Boulnois, 2001). Après cet Age d'or, la région sombrera dans un isolement profond et durable à partir du $\mathrm{XVI}^{\mathrm{e}}$ siècle avec la généralisation du transport maritime et l'insécurité liée aux troubles politiques.

3 Mais depuis peu l'Asie centrale réapparaît dans le champ des observateurs internationaux et des géographes à la faveur des retournements géopolitiques qui ont affecté les deux grandes puissances communistes. En premier lieu, la disparition brutale de l'URSS en 1991 a entraîné la création de cinq républiques indépendantes, faisant ainsi entrer l'Asie centrale ex-soviétique dans un processus de décolonisation. A l'opposé, l'ouverture de la République populaire de Chine à l'économie de marché dans les années 1980 et la mise en place du Programme de développement de l'Ouest chinois en 2000, ont accéléré le processus de colonisation du Xinjiang amorcé depuis 1949. Indépendances et décolonisation difficile côté Asie centrale post-soviétique, intensification de la sinisation et montée du séparatisme ouïgour côté chinois, tout concours à faire de la région un espace en recomposition. C'est donc un nouveau cycle historique qui s'amorce où la redéfinition des équilibres politiques s'accompagne de multiples dynamiques géographiques dont certaines sont abordées dans ce dossier. Pour les jeunes républiques nées de l'implosion de l'URSS, l'heure est à la construction et à l'affirmation de nouveaux Etats-nations (Laruelle et Peyrouse, 2006). Celles-ci tentent de rompre avec l'isolement dans lequel elles ont été durablement confinées par l'ancienne puissance tutélaire en s'ouvrant sur la scène internationale. Leur intégration dans le marché mondial se fait par l'exportation de matières premières, principalement les hydrocarbures, l'or, l'uranium et le coton dont la rente est le plus souvent accaparée au profit des pouvoirs politiques à la tête d'Etats autoritaires. Au Xinjiang, le gouvernement chinois travaille activement à l'intégration économique et culturelle d'une périphérie constituée de minorités nationales turcophones et par ailleurs riche en ressources naturelles. La sinisation de la région autonome vise à sécuriser cette marge jugée turbulente, à en exploiter les matières premières énergétiques et à l'ouvrir sur l'Asie centrale post-soviétique où la Chine entend bien occuper la place laissée vacante par la disparition de l'URSS.

Cette nouvelle situation géopolitique s'avère particulièrement fertile pour les géographes soucieux de saisir et de comprendre les évolutions qui affectent l'ensemble de l'environnement géographique centrasiatique. Ainsi dans son article Guillemette Pincent montre comment les Etats utilisent le patrimoine urbain comme un instrument politique. Si durant la période soviétique les bâtiments religieux d'Asie centrale soviétique étaient détruits ou détournés de leur fonction afin de répondre aux préceptes de l'idéologie communiste, aujourd'hui les jeunes républiques indépendantes ne se soucient guère de protéger les édifices de la période coloniale et soviétique qui disparaissent progressivement $d u$ paysage urbain. En revanche les monuments historiques de la période précoloniale font l'objet d'une attention particulière dans la mesure où ils constituent des symboles architecturaux propres à servir l'idéologie post- 
soviétique. Ainsi en Ouzbékistan, la mise en valeur et la restauration portent particulièrement sur les monuments historiques de la période Timouride (XIV-XVI ${ }^{\mathrm{s}} \mathrm{s}$ ) qui témoignent du prestige et de la puissance des souverains centrasiatiques d'alors. Ces choix sélectifs doivent permettre la construction d'une mémoire collective et d'un passé commun et servent de justification historique à des Etats qui n'ont pas plus de vingt ans d'existence. Au Xinjiang, les vieilles villes aux allures de médina, symbole culturel de la minorité nationale ouïgoure, sont détruites par pans entiers, remplacées par un nouveau modèle urbain synonyme de "modernisation" et de sinisation. Quelques quartiers et édifices historiques comme les grandes mosquées sont néanmoins conservés mais dans un but de valorisation touristique. Ainsi la ville centrasiatique détruite, transformée, reconstruite se recompose au gré des valeurs politiques et de l'idéologie que cherchent à faire triompher les différents régimes autoritaires qui se partagent la région.

Un autre phénomène géographique remarquable concerne l'évolution des populations. Dans son article consacré à l'émigration des russophones, Julien Thorez analyse pourquoi et comment l'Asie centrale post-soviétique est devenue une terre d'émigration et s'inscrit dans le système migratoire mondial. Si durant la période tsariste et soviétique la région a connu un excédent migratoire dû à la politique coloniale et à son rôle de terre de relégation pour les populations et les peuples punis, la situation actuelle témoigne d'une inversion des dynamiques migratoires. Depuis 1990 près de 5 millions de personnes, pour l'essentiel des Russes, ont quitté l'Asie centrale post-soviétique ; ce qui tend à renforcer l'homogénéité ethnique de chaque Etat où la proportion de la nationalité éponyme s'accroît sensiblement. Le départ des russophones est étudié sous l'angle de la crise économique et de l'altération de leur statut social et politique sous l'effet de la construction et de l'affirmation des Etats et des nations post-soviétiques.

6 L'Asie centrale chinoise renvoie quant à elle à une situation démographique et historique inverse. En effet l'article consacré au Xinjiang montre que ce territoire constitue une terre d'immigration dans le cadre de la politique d'intégration et de sinisation de l'Ouest chinois menée par le gouvernement central. L'actuelle situation de la région autonome illustre bien ce concept de «nouvelle frontière » dans la mesure où les flux de migrants Han investissent et organisent progressivement ce territoire traditionnellement occupé par des populations locales turcophones qualifiées de minorités nationales. C'est à la faveur de l'essor d'un réseau ferroviaire et routier moderne que se diffuse la sinisation créatrice de corridors de développement où se multiplient les fronts pionniers agricoles, industriels et urbains fondés sur la valorisation des ressources naturelles. Il en résulte un bouleversement des équilibres ethniques où les minorités nationales sont en passe d'être minoritaires sur leur propre territoire. Avec la maîtrise de cette périphérie, la Chine entend faire du Xinjiang une tête de pont stratégique permettant la diffusion de l'influence chinoise au cœur de l'Asie. Après l'ouverture de sa façade littorale, la Chine entrebâille une nouvelle fenêtre, continentale cette fois, sur son hinterland centrasiatique.

7 En définitive, l'évolution des grands équilibres géostratégiques au cours des deux dernières décennies a projeté l'Asie centrale dans une nouvelle ère de transition synonyme de turbulences et de changements. Que ce soit la renaissance politique et identitaire de l'Asie centrale post-soviétique ou l'intégration politique et l'assimilation culturelle de l'Asie centrale chinoise, les deux trajectoires, pour le moins opposées, 
témoignent cependant d'une recomposition profonde qui affecte tous les champs de la géographie ce qu'attestent les regards de géographes présentés dans ce dossier.

\section{BIBLIOGRAPHIE}

Aubin F., 1990, Donneurs ou preneurs, quelques réflexions sur le jeu des transferts culturels entre Chinois, Turcs et Mongols, in Dor R. L'Asie centrale et ses voisins, Inalco, Paris, p. 165-180.

Boulnois L., 2001, La Route de la Soie. Dieux, Guerriers et Marchands, Editions Olizane, Genève, 558 p.

Cariou A., 2007, L'Asie centrale dans la mondialisation : de la marginalisation à l'intégration, in Cadène P., La mondialisation. L'intégration des pays en développement, SEDES, Paris, p. 163-174.

Chuvin P., Létolle R., Peyrouse S., 2008, Histoire de l'Asie centrale contemporaine, Fayard, Paris, 376 p.

Kellner T., 2008, L'Occident de la Chine. Pékin et la nouvelle Asie centrale (1991-2001), Presses Universitaires de France, Paris, 622 p.

Laruelle M., Peyrouse S., 2006, Asie centrale, la dérive autoritaire. Cinq républiques entre héritage soviétique, dictature et islam, CERI-Autrement, Paris, $135 \mathrm{p}$.

Peyrouse S. (dir.), 2004, Gestion des indépendances et legs soviétique en Asie centrale in Cahiers d'Asie centrale, $\mathrm{n}^{\circ}$ 13-14, Iféac-Edisud, $334 \mathrm{p}$.

Radvanyi J. (dir.), 2003, Les Etats postsoviétiques. Identités en construction, transformations politiques, trajectoires économiques, Paris, Armand Colin, $235 \mathrm{p}$.

Sellier J. et Sellier A, 2007, Atlas des peuples d'Orient. Moyen-Orient, Caucase, Asie centrale, La Découverte, Paris, 206 p.

Roy O., 1997, La Nouvelle Asie centrale ou la fabrication des nations, Seuil, Paris, 326 p. 